\title{
Protective effects of valsartan administration on doxorubicin-induced myocardial injury in rats and the role of oxidative stress and NOX2/NOX4 signaling
}

\author{
DONG CHENG ${ }^{1,2 *}$, LIBO CHEN $^{1,3 *}$, WENCHENG TU $^{1,4^{*}}$, HAOREN WANG $^{5}$, \\ QINFU WANG ${ }^{6}$, LILI MENG ${ }^{1,7}, \mathrm{ZHU}^{1,8}$ and QIN YU ${ }^{1}$ \\ ${ }^{1}$ Department of Cardiology, Affiliated Zhongshan Hospital of Dalian University, Dalian, Liaoning 116001; \\ ${ }^{2}$ Medical College, Dalian University, Dalian, Liaoning 116622; ${ }^{3}$ Department of Cardiology, People's Hospital of Jilin City, \\ Changchun, Jilin 132000; ${ }^{4}$ Department of Cardiology, Jingmen No. 1 People's Hospital, Jingmen, Hubei 448000; \\ ${ }^{5}$ Central Laboratory, Affiliated Zhongshan Hospital of Dalian University, Dalian, Liaoning 116001; ${ }^{6}$ Life Engineering College, \\ Dalian University, Dalian, Liaoning $116622 ;{ }^{7}$ Emergency Internal Medicine, Jining No. 1 People's Hospital, Jining, \\ Shandong 272000; ${ }^{8}$ Department of Cardiology, Zhuanghe Central Hospital, Dalian, Liaoning 116000, P.R. China
}

Received November 27, 2019; Accepted July 14, 2020

DOI: $10.3892 / \mathrm{mmr} .2020 .11521$

\begin{abstract}
Clinical application of doxorubicin (DOX) is hampered by its potential cardiotoxicity, however angiotensin receptor blockers could attenuate DOX-induced cardiomyopathy. The present study tested the hypothesis that simultaneous administration of valsartan (Val) with DOX could prevent DOX-induced myocardial injury by modulating myocardial $\mathrm{NAD}(\mathrm{P}) \mathrm{H}$ oxidase (NOX) expression in rats. Eight-week-old male Sprague-Dawley rats were randomly divided into control (CON), DOX, and DOX+Val groups. After 10 weeks, surviving rats underwent echocardiography examination, myocardial mRNA and protein expression detection of NOX1, NOX2 and NOX4. H9C2 cells were used to perform in vitro experiments, reactive oxygen species (ROS) production and apoptosis were observed under the conditions of down- or upregulation of NOX2 and NOX4 in DOX- and DOX+Val-treated H9C2 cells. Cardiac function was significantly improved, pathological lesion and collagen volume fraction were significantly reduced in the DOX+Val group compared with the DOX group (all $\mathrm{P}<0.05$ ). Myocardial protein and mRNA expression of NOX2 and NOX4 was significantly downregulated in DOX+Val group compared with in the DOX group (all $\mathrm{P}<0.05)$. In vitro, ROS production and apoptosis in DOX-treated $\mathrm{H} 9 \mathrm{C} 2$ cells was significantly reduced by
\end{abstract}

Correspondence to: Professor Qin Yu, Department of Cardiology, Affiliated Zhongshan Hospital of Dalian University, 6 Jiefang, Zhongshan, Dalian, Liaoning 116001, P.R. China

E-mail: yuqin@dlu.edu.cn

${ }^{*}$ Contributed equally

Key words: angiotensin receptor blocker, chemotherapy, NAD(P)H oxidase, oxidative stress, apoptosis
NOX2-small interfering (si)RNA and NOX4-siRNA, and significantly increased by overexpressing NOX2 and NOX4. To conclude, Val applied simultaneously with DOX could prevent DOX-induced myocardial injury and reduce oxidative stress by downregulating the myocardial expression of NOX2 and NOX4 in rats.

\section{Introduction}

Doxorubicin (DOX) is highly effective treatment against acute lymphoblastic and myeloblastic leukemia, and numerous types of solid tumors, including breast cancer, sarcomas and childhood solid tumors (1). However, clinical use of DOX is hampered by its potential cardiotoxicity (1-5). Angiotensin receptor blockers (ARBs) may attenuate DOX-induced cardiomyopathy (6-9); however, the underlying mechanisms are not fully understood and there are very few studies examining the effects of simultaneous treatment with DOX and ARBs for the prevention of DOX-induced myocardial injury. Valsartan (Val), which is a type of ARB, is widely used to treat patients with hypertension and heart failure (10). A clinical observational study demonstrated that Val could prevent the acute cardiotoxicity induced by cyclophosphamide, doxorubicin, vincristine and prednisolone, which are standard chemotherapeutic options for treatment of non-Hodgkin lymphoma (11). More recently, Sakr et al (12) investigated the effect of Val on DOX-induced cardiotoxicity in rats, and found that concurrent or post-but not pre-treatment with Val attenuated DOX-induced cardiotoxicity by inhibiting oxidative stress, apoptosis and senescence. Another study reported that Val alleviated DOX-induced cardiac dysfunction via regulation of the TGF- $\beta$ signaling pathway (13).

Excessive reactive oxygen species (ROS) production is a known risk factor responsible for the initiation and development of heart failure $(14,15)$ and DOX-induced cardiotoxicity. $\mathrm{NAD}(\mathrm{P}) \mathrm{H}$ oxidase (NOX) is one of several contributing sources responsible for increased ROS generation (16). NOX 
may be activated by growth factors or inflammatory cytokines (17-19). Previous studies confirmed that angiotensin II may stimulate ROS production by activating NOX (20-23). Therefore, it may be hypothesized that reduced NOX and ROS signaling is involved in the beneficial effects of ARB on attenuating DOX-induced cardiotoxicity.

The aim of the present study was to test the hypothesis that simultaneous treatment with Val could prevent DOX-induced myocardial injury by downregulating myocardial NOX expression and reducing ROS production in rats (Fig. 1).

\section{Materials and methods}

Reagents. DOX was purchased from Zhejiang Hisun Pharmaceutical Co., Ltd. Val was purchased from Beijing Novartis Pharmaceutical Co., Ltd. (https://www.novartis.com.cn/).

Animal model and study protocol. A total of 40 specific pathogen free-grade 8-week-old male Sprague-Dawley rats (body weight, 200-250 g) were purchased from the Experimental Animal Center of Dalian Medical University. Rats were housed in $530 \mathrm{~cm}^{2}$ cages with wood-shaving bedding ( 2 rats per cage) in a temperature-controlled room $\left(25 \pm 2^{\circ} \mathrm{C}\right)$, the humidity was maintained at $50-70 \%$, the noise was $<85$ decibels, with $14 \mathrm{~h}$ of light and $10 \mathrm{~h}$ of darkness every day. Rats were fed under standard conditions with free access to food and drinking water. Rats were randomly divided into three groups: i) Control group ( $\mathrm{CON}, \mathrm{n}=8)$, rats treated with equal volume saline daily via gavage for 6 weeks; ii) DOX group $(\mathrm{n}=18)$ rats received intraperitoneal DOX $(2.5 \mathrm{mg} / \mathrm{kg})$ injection once per week for 6 weeks; iii) DOX+Val group $(n=14)$, rats received intraperitoneal DOX $(2.5 \mathrm{mg} / \mathrm{kg})$ injection once per week plus Val $(20 \mathrm{mg} / \mathrm{kg})$ daily via gavage for 6 weeks. After another 4 weeks, surviving rats underwent echocardiography examination. Subsequently, rats were sacrificed under deep anesthesia (intramuscular ketamine hydrochloride injection, $100 \mathrm{mg} / \mathrm{kg}$ ), and the heart was isolated and weighed. The atria and right ventricle were separated from the left ventricle (LV), and the LV was cut into three sections along the LV long-axis at a thickness of $3 \mathrm{~mm}$. The middle section of the LV was processed for histological examination. The basal and the apical sections were stored at $-80^{\circ} \mathrm{C}$ for immunohistological and biochemical analysis. The experimental protocol is presented in Fig. 1. All experiments were performed in compliance with the ARRIVE guidelines as well as the Guide for the Care and Use of Laboratory Animals of the National Academy of Sciences. The Institutional Animal Research and Ethics Committee of Dalian University approved the protocols of the animal experiments.

Echocardiography. Under light anesthesia (intramuscular ketamine hydrochloride injection, $22 \mathrm{mg} / \mathrm{kg}$ ), rats underwent echocardiography using a Vivid E9 dimension system (General Electric Company), equipped with a $12.0 \mathrm{MHz}$ transducer. Two-dimensional and M-mode echocardiography images were obtained in the parasternal long-axis and short-axis views of the heart. All measurements were performed online with optimal images from $>10$ cardiac cycles taken by an experienced sonographer who was blinded to the study protocol and grouping (24). Left ventricular end-diastolic (LVEDD) and end-systolic diameters (LVESD) were measured with M-mode in the parasternal short-axis images at the papillary muscle level. Left ventricular fractional shortening (LVFS) was calculated as follows: LVFS (\%) = (LVEDD-LVESD)/LVEDD x 100. Left ventricular ejection fraction (LVEF) was calculated according to the Teichholz formula (25).

Histopathological evaluation. The LV tissue assigned for histological examination (middle section) was fixed in $4 \%$ paraformaldehyde for $24 \mathrm{~h}$ at $4^{\circ} \mathrm{C}$, embedded in paraffin, sectioned into $5 \mu \mathrm{m}$ slices and stained with Masson (Shanghai Bogoo Biotechnology Co., Ltd.; http://www.bgswkj.com/) stain A for $15 \mathrm{~min}$ and stain B for $20 \mathrm{~min}$. Then, slices were stained with Picrosirius Red (Beijing Solarbio Science $\&$ Technology Co., Ltd.) for $1 \mathrm{~h}$ at room temperature and observed with a light microscope (magnification, x100). Interstitial collagen volume fraction (CVF) was determined using Image-Pro Plus 6.0 (Media Cybernetics, Inc.). CVF was calculated as follows: $\mathrm{CVF}(\%)=$ area of stained collagen $/$ total area of field of vision $\mathrm{x} 100$.

ELISA. Specimens from left ventricular tissues of rats in each group were weighed and cut into pieces, mixed with pre-cooled PBS at a ratio of 1:9 (weight:volume) to prepare the tissue homogenate. Myocardial levels of renin (Sigma-Aldrich; Merck KGaA; cat. no. RAB1162), TNF- $\alpha$ (Sigma-Aldrich; Merck KGaA; cat. no. RAB0479), IL-6 (Sigma-Aldrich; Merck KGaA; cat. no. RAB0311), brain natriuretic peptide (BNP; Sigma-Aldrich; Merck KGaA; cat. no. RAB0386), aldosterone (ALD; Abcam; cat. no. Ab136933), malondialdehyde (MDA; Abcam; cat. no. Ab238537), ROS (Jianglai Bio; http://www.laibio.com/; cat.no.JL21051), superoxide dismutase (SOD; Jianglai Bio; cat. no. JL11065), NOX1 (Jianglai Bio; cat. no. JL36449), NOX2 (Jianglai Bio; cat. no. JL50110) and NOX4 (Jianglai Bio; cat. no. JL23194) were measured. ELISA was performed according to the manufacturer's protocol and a DNA Eraser was used (Takara Bio, Inc.).

Reverse transcription-quantitative $(R T-q) P C R$. All primer sequences were designed and synthesized by Sangon Biotech Co., Ltd., which are presented in Table I. PrimeScript ${ }^{\mathrm{TM}}$ RT Reagent kit with a gDNA Eraser (Takara Bio, Inc.) was used for reverse-transcription at $37^{\circ} \mathrm{C}$ for $15 \mathrm{~min}$ and $85^{\circ} \mathrm{C}$ for 5 sec. qPCR was performed using SYBR ${ }^{\circledR}$ Premix Ex Taq ${ }^{\mathrm{TM}}$ II (Takara Bio, Inc.). qPCR was performed as described previously (26). Briefly, qPCR was performed in $20 \mu \mathrm{l}$ reaction containing $10 \mu \mathrm{l} \mathrm{SYBR}$ Premix Ex Taq II (Tli RNaseH Plus), Bulk, $0.8 \mu \mathrm{l}$ of each primer $(10 \mu \mathrm{M}), 0.4 \mu \mathrm{l}$ ROX Reference

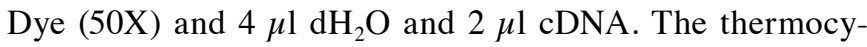
cling conditions were: Pre-denaturation for $30 \mathrm{sec}$ at $95^{\circ} \mathrm{C}$; followed by 30 cycles of $95^{\circ} \mathrm{C}$ for $5 \mathrm{sec}$ and $60^{\circ} \mathrm{C}$ for $30 \mathrm{sec}$. Relative quantities of all targets in test samples were normalized to the respective GAPDH levels. $2^{-\Delta \Delta \mathrm{Cq}}$ was calculated as follows (27): $\Delta \mathrm{Cq}=\mathrm{DOX}$ group (target gene $\mathrm{Cq}$ value-made in $\mathrm{Cq}$ )-control group (target gene $\mathrm{Cq}$ value-made in $\mathrm{Cq}$ value). For different groups of the genes, the internal change ratio $=2^{-\Delta \Delta \mathrm{Cq}}$. The expression levels were estimated using the integrated optical density (OD) of the positive cells. Integrated OD was the average cumulative OD of the positive staining area of each group determined by Image Pro Plus version 6.0 

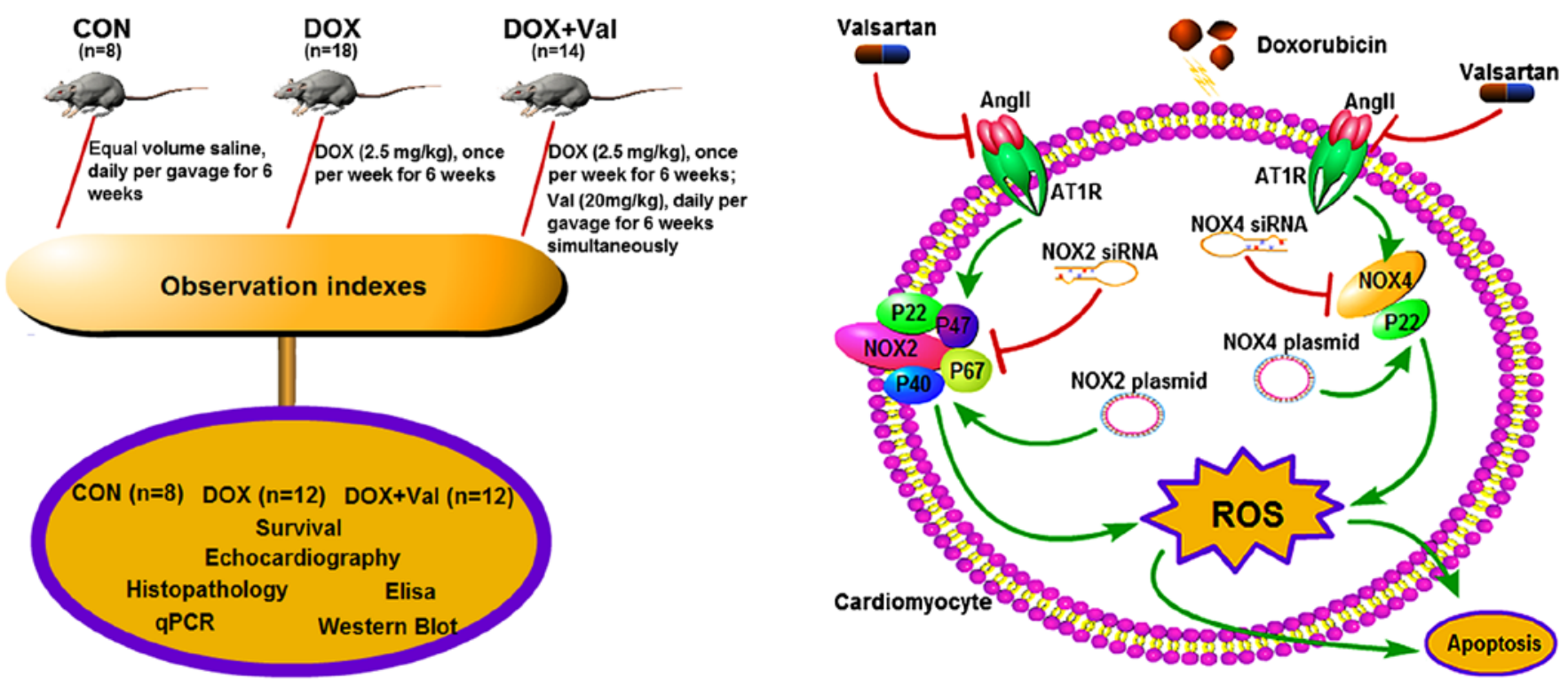

Figure 1. Schematic diagram of experiments. CON, control; DOX, doxorubicin; Val, valsartan.

(Media Cybernetics, Inc.). RT-qPCR experiments were performed in triplicate.

Western blot analysis. Myocardial protein expression levels of NOX1, NOX2 and NOX4 were determined by western blotting as described previously (28). Extracted membrane proteins from LV tissue was quantified using a BCA protein assay kit and protein $(25 \mu \mathrm{g} /$ lane $)$ was separated via SDS-PAGE on a $20 \%$ gel, and subsequently transferred to a polyvinylidene difluoride membrane. Then, the membrane was blocked with 5\% BSA blocking buffer (Nanjing KeyGen Biotech Co., Ltd.) at room temperature for $1 \mathrm{~h}$, and incubated overnight at $4^{\circ} \mathrm{C}$ with rabbit anti-human NOX1 (1:5,000; cat. no. GTX103888), rabbit anti-human NOX2 (1:5,000; cat. no. GTX133715) and rabbit anti-human NOX4 antibodies (1:1,000; cat. no. GTX121929; all purchased from GeneTex, Inc.), and the loading control rabbit anti-human $\mathrm{Na}^{+} / \mathrm{K}^{ \pm}$ATPase $\alpha-1$ antibodies $(1: 10,000$; cat. no. ab76020; Abcam), followed by secondary goat anti-rabbit IgG (1:5,000; cat. no. ab6721; Abcam) or at room temperature for $1.5 \mathrm{~h}$. The signal was developed by applying goat anti-rabbit IgG conjugated to horseradish peroxidase, and thereafter exposed to X-ray films that were scanned and determined by ImageJ software v1.8.0 (National Institutes of Health) to quantify protein expression.

\section{In vitro experiments}

Cell culture. H9C2 cardiomyocytes (China Center for Type Culture Collection) were maintained at $37^{\circ} \mathrm{C}$ in a humidified incubator with $5 \% \mathrm{CO}_{2}$. Cells were cultured in DMEM (Gibco; Thermo Fisher Scientific, Inc.) supplemented with $10 \%$ FBS (AusGeneX, Ltd.), $100 \mathrm{U} / \mathrm{ml}$ penicillin and $100 \mu \mathrm{g} / \mathrm{ml}$ streptomycin, $0.25 \%$ trypsin and $0.02 \%$ EDTA digestive solution were used to trypsinize the cells. After the third passage, $\mathrm{H} 9 \mathrm{C} 2$ cells were harvested for further experiments.

Downregulation of NOX2 and NOX4 via small interfering RNA (siRNA) transfection. siRNA targeting NOX2 $(20 \mu \mathrm{M}$; $10 \mu \mathrm{l} /$ well), siRNA targeting NOX4 $(20 \mu \mathrm{M} ; 10 \mu \mathrm{l} /$ well $)$ and the negative random siRNA $(20 \mu \mathrm{M} ; 10 \mu \mathrm{l} /$ well; Shanghai GenePharma Co., Ltd.) were transiently transfected into H9C2 cells (1x10 $/$ well) using Lipofectamine ${ }^{\circledR} 2000$ (Thermo Fisher Scientific, Inc.), according to the manufacturer's protocols and a previous study (29). The sequences of the siRNAs were as follows: NOX2-rat-663 siRNA sense, 5'-CCAUGGAGCUGA ACGAAUUTT-3' and anti-sense, 5'-AAUUCGUUCAGCUCC AUGGTT-3'; and NOX4-rat-576 siRNA sense, 5'-GCUUCU ACCUAUGCAAUAATT-3' and anti-sense, 5'-UUAUUGCAU AGGUAGAAGCTT-3'.

Upregulation of NOX2 and NOX4 via construction and transfection of eukaryotic overexpression plasmids. Total RNA was extracted from cultured H9C2 cells using TRIzol reagent (Invitrogen; Thermo Fisher Scientific, Inc.). cDNA was synthesized and the PCR product was inserted into a pMD18-T plasmid (Takara Bio, Inc.). Insertion was confirmed using the restriction enzymes XhoI and $K p n I$ (Takara Bio, Inc.) and DNA sequencing. The NOX2 and NOX4 genes were cloned into a pEX-4 (pGCMV/MCS/T2A/EGFP/Neo) vector (Shanghai GenePharma Co., Ltd.). Recombinant plasmids were obtained and identified by digestion with XhoI and KpnI. The following primers were designed with specific restriction enzyme sites to clone the complete coding region of NOX2 and NOX4: NOX2 forward, 5'-GCGCTACCGGACTCAG ATCTCGAGGCCACCATGGGGAACTGGGCTGTGAATG AGGGACTC-3' (XhoI) and reverse, 5'-ACTTCCTCTGCC CTCGGTACCGAAGTTTTCCTTGTTGAAGATGAAGTGG ACTCCACGTGG-3' (KpnI); and NOX4 forward, 5'-GCTACC GGACTCAGATCTCGAGGCCACCATGGCGCTGTCCTGG AGGAGCTGGCTGGCCAA-3' (XhoI) and reverse, 5'-ACT TCCTCTGCCCTCGGTACCGCTGAAAGATTCTTTATTG TATTCAAATTTTGTCCCATA-3' (KpnI). The optimized PCR amplification conditions were: Annealing at $58^{\circ} \mathrm{C}$ and extension at $72^{\circ} \mathrm{C}$ for 35 cycles. H9C 2 cells $\left(1 \times 10^{5} /\right.$ well) were transfected with pEX-4-NOX2/NOX4 $(1 \mu \mathrm{g} / \mu \mathrm{l} ; 2.5 \mu \mathrm{l} /$ well $)$ or empty vectors $(1 \mu \mathrm{g} / \mu \mathrm{l} ; 2.5 \mu \mathrm{l} / \mathrm{well})$, which was added to balance the total amount of transfected DNA using 
Table I. Primer sequences for reverse transcription-quantitative PCR.

Gene Primer sequence $\left(5^{\prime} \rightarrow 3^{\prime}\right)$

Bax

Collagen I F: CGGTGGTTATGACTTCAG CTTC

F: GTGGTTGCCCTCTTCTACTTTG R: CCACAAAGATGGTCACTGTCTG R: AGAGGGCTGAGTGGGGAAC

BNP F: GCAGGCCAGCAGGGTCCACTACAC R: GACCTC GCCATTCCGCTGATTCT

Beclin-1 F: GACAAATCTAAGGAGTTGCCG R: AGAACTGTGAGGACACCCA

BCL2 F: AGTTCGGTGGGGTCATGTGTG R: CCAGGTATGCACCCAGAGTG

NOX4 F: GAACCCAAGTTCCAAGCTCA R: GCA CAAAGGTCCAGAAATCC

NOX1 F: GGCATCCCTTTACTCTGACCT R: TGCTGCTCGAATATGAATG

NOX2 F: TGTGCACCACGATGAGGAGAAAGATG R: ATTCTGGTGTTGGGGTGTGACTFGC

Caspase-3 F: CTTTGCGCCATGCTG AAACT R: TCAAATTCCGTGGCCACTT

MMP9 F: TCCCCAGAGCGTTACTCG CT R: ACCTGG TTCACCCGGTTGTG

MMP2 F: GAGTAAGAACAAGAAGACATACATC R: GTAATAAGCACCCTTGAA GAAATAG

ANP F: GGGTGTGAACCA CGAGAAAT R: ACTGTGGTCATGAGCCCTTC

$\beta$-MHC F: GGGCAA AGGCAAAGCAAAGA R: AAAGTGAGGGTGCGTGGAGC

BGN F: ACAACAAGCTGTCCCGGGTG R: AGC CCATGGGGCAGAAATCG

TPM1 F: GCTGGTTGAGGAGGAGTT R: ACTTGTTCGTCACCGTTT

Collagen III F: GGAGTCGGAGGAATGGGTG R: ATTGCGTCCATCAAAGCCTC CORIN F: GTACAG TGCGGTGTCCAACA R: ATCCTGTCAATCCTACCCCC

TGF- $\beta 1 \quad F$ : CCAACTATTGCTTCAGCTCCA R: GTGTCCAGGCTCCAAATGT

GDF15 F: CTGCTCTTGCTGCTTCTGCT R: TCGTCCGGGTTGAGTTGG

POSTN F: AGGAGCCGTGTTTGAGACCAT R: CGGTGAAAGTGGTTT GCTGTTT

GAPDH F: GGTGCTGAGTATGTCGTGGAGT R: CACAGT CTTCTGAGTGGCAGTG

F, forward; R, reverse; NOX, NAD $(\mathrm{P}) \mathrm{H}$ oxidase; BNP, brain natriuretic peptide; $\mathrm{MMP}$, matrix metallopeptidase; $\mathrm{ANP}$, atrial natriuretic peptide; $\beta$-MHC, $\beta$ myosin heavy chain; GDF15, growth differentiation factor 15; TPM1, tropomyosin 1; BGN, biglycan; POSTN, periostin; CORIN, atrial natriuretic peptide-converting enzyme; TGF- $\beta 1$, transforming growth factor $\beta 1$.
Lipofectamine 2000 according to the manufacturer's protocols and a previous study (30). Untransfected cells were used as controls. Cells were cultured for $24 \mathrm{~h}$ before use in subsequent experiments.

Cell viability. H9C2 cells $\left(3 \times 10^{4} /\right.$ well) were treated with DOX $(0.1,0.3,0.5,1,3,5,10,30$ or $50 \mu \mathrm{M})$ and $\operatorname{Val}(0.1,0.5,1,3,5$, $7.5,10,15$ or $30 \mu \mathrm{M}$ ) for $12,24,48$ or $72 \mathrm{~h}$ ) with $5 \% \mathrm{CO}_{2}$, at $37^{\circ} \mathrm{C}$ in an incubator, and subsequently harvested for further molecular and biochemical analyses. Untreated H9C2 cells and DMSO-pre-treated H9C2 cells were used as the control groups. All in vitro experiments were performed in triplicate. Cell viability was determined using a modified MTT assay as described previously (31). Briefly, MTT solution in PBS $(5 \mathrm{mg} / \mathrm{ml})$ was added to each well at a final concentration of $0.05 \%$. After $3 \mathrm{~h}$, the formazan precipitate was dissolved in DMSO. The absorbance was measured at 570 and $620 \mathrm{~nm}$ (background) using Epoch ${ }^{\mathrm{TM}}$ microplate spectrophotometer (BioTek Instruments, Inc.).

In vitro measurement of intracellular ROS production. To measure intracellular ROS production, H9C2 cells $\left(6 \times 10^{4} /\right.$ well $)$ were seeded in a 24 -well culture plate and incubated with dichlorofluorescein diacetate (DCFH-DA; $10 \mathrm{mM}$; Sigma-Aldrich; Merck KGaA) for $1 \mathrm{~h}$ at $37^{\circ} \mathrm{C}$ in the dark. After the various aforementioned treatments, cells were washed immediately and resuspended in PBS solution. Viable cells incorporate 2',7'-DCFH-DA, which is not fluorescent, but in the presence of ROS, DCFH-DA reacts with oxygen species to produce the fluorescent dye 2',7'-DCF. Fluorescence emission was measured by flow cytometry (BD FACSCanto ${ }^{\mathrm{TM}}$ II Flow Cytometer; BD Biosciences) using a $525 \mathrm{~nm}$ band pass filter, which provides an index of the intracellular oxidative metabolism (32). ROS levels were detected by performing a DCFH assay and semi-quantitative analysis (BD FACSDiva software; version 7.0; BD Biosciences). The cells were divided into ten groups and treated as follows: i) Control group included $\mathrm{H} 9 \mathrm{C} 2$ cells without any treatment; ii) $\mathrm{H} 9 \mathrm{C} 2$ cells exposed to $1 \mu \mathrm{M}$ DOX for $24 \mathrm{~h}$; iii) cells exposed to $5 \mu \mathrm{M} \mathrm{Val}$ for $1 \mathrm{~h}$; iv) cells pre-treated with $5 \mu \mathrm{M}$ Val for $1 \mathrm{~h}$ followed by treatment with $1 \mu \mathrm{M}$ DOX for $24 \mathrm{~h}$; v) cells transiently transfected with NOX2-siRNA for $24 \mathrm{~h}$ followed by treatment with $1 \mu \mathrm{M}$ DOX for $24 \mathrm{~h}$; vi) cells transiently transfected with NOX4-siRNA for $24 \mathrm{~h}$ followed by treatment with $1 \mu \mathrm{M}$ DOX for $24 \mathrm{~h}$; vii) cells transiently transfected with negative siRNA for $24 \mathrm{~h}$ followed by treatment with $1 \mu \mathrm{M}$ DOX for $24 \mathrm{~h}$; viii) cells transiently transfected with an empty vector for $24 \mathrm{~h}$; ix) NOX2-overexpressing cells treated with $1 \mu \mathrm{M}$ DOX for $24 \mathrm{~h}$; and $\mathrm{x}$ ) NOX4-overexpressing cells were treated with $1 \mu \mathrm{M}$ DOX for $24 \mathrm{~h}$.

Apoptosis and flow cytometry. Cardiomyocyte apoptosis was evaluated using flow cytometry and DNA electrophoresis. A total of $1 \times 10^{5} \mathrm{H} 9 \mathrm{C} 2$ cells/well were seeded in 12-well culture plates and cardiomyocytes under various treatments were trypsinized, washed with PBS solution, centrifuged at $800 \mathrm{xg}$ for $6 \mathrm{~min}$ at $4^{\circ} \mathrm{C}$, resuspended in ice-cold $70 \%$ ethanol/PBS, centrifuged at $800 \mathrm{x} \mathrm{g}$ for another $6 \mathrm{~min}$ at $4^{\circ} \mathrm{C}$, and resuspended in PBS. Cells were then incubated with propidium iodide (PI) and FITC-labeled Annexin V for $30 \mathrm{~min}$ at $37^{\circ} \mathrm{C}$. 

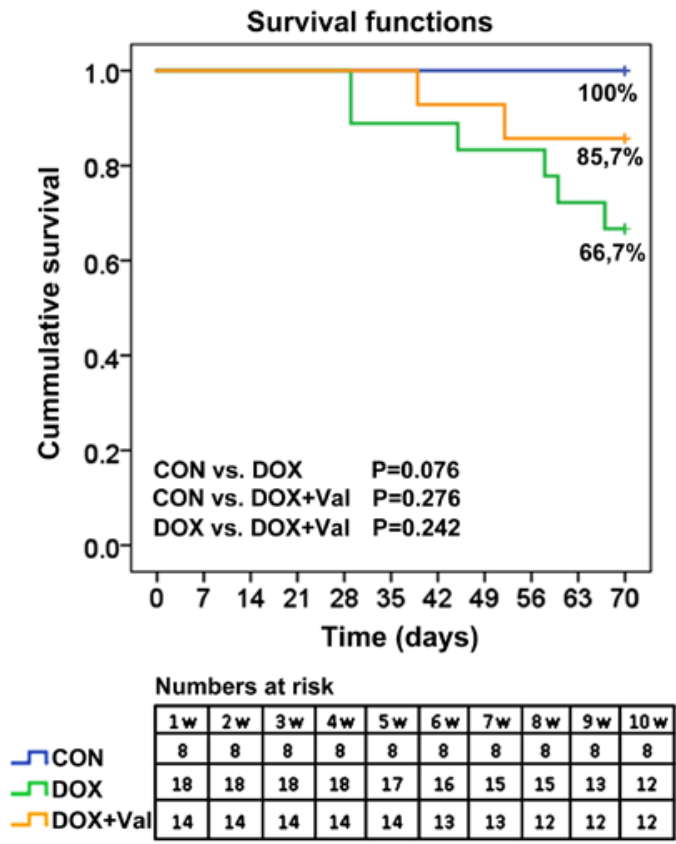

Figure 2. Cumulative survival rates of rats post-treatment in each experimental group. CON, control; DOX, doxorubicin; Val, valsartan.

Cells were washed to remove excess PI and Annexin V, and then analyzed by flow cytometry using a FACSCalibur ${ }^{\mathrm{TM}}$ flow cytometer (BD Biosciences) with a $488 \mathrm{~nm}$ argon laser light source, a $525 \mathrm{~nm}$ band pass filter for FITC fluorescence, and a $625 \mathrm{~nm}$ band pass filter for PI-fluorescence. Flow cytometry data were analyzed using CellQuest software version 3.0 (BD Biosciences). Dot plots of PI fluorescence (y-axis) vs. FITC fluorescence (x-axis) are presented. The assessment of apoptosis rate was the sum of early and late apoptosis. Apoptosis analysis was performed in the aforementioned ten groups that were used to measure intracellular ROS production.

Statistical analysis. Data are presented as the mean \pm standard deviation and were analyzed using one-way or two-way ANOVA followed by Bonferroni's pos hoc comparisons using SPSS software (version 20.0; IBM Corp.). Kaplan-Meier curves were used to plot and estimate survival. $\mathrm{P}<0.05$ was considered to indicate a statistically significant difference.

\section{Results}

Cumulative survival rates of rats. All rats $(n=8)$ in the CON group survived to the end of the study. In the DOX group, 16 of the $18(88.9 \%)$ rats were alive at week 7 and $12(66.7 \%)$ rats were alive at week 10 . In the DOX+Val group, 13 of the $14(92.9 \%)$ rats were alive at week 6 and $12(85.7 \%)$ rats were alive at week 10 (Fig. 2).

Effect of DOX+Val on LV function and CVF. At week 10 of the study, LVEF and LVFS were significantly lower, whereas LVESD was significantly higher in the DOX group compared with the CON group. LVEF and LVFS were significantly higher and LVESD was significantly lower in the DOX+Val group compared with the DOX group (Fig. 3).
Interstitial CVF values measured using Masson staining and Sirius Red staining were significantly higher in the DOX group compared with the CON group. In the DOX+Val group, the values were significantly lower compared with the DOX group (Fig. 4A and B). Overall, these results suggest that simultaneous application of Val with DOX attenuated DOX-induced myocardial injury in rats, as shown by improved cardiac function and attenuated cardiac remodeling.

Effect of DOX+Val on myocardial mRNA expression levels of various signaling molecules. The myocardial mRNA expression levels of NOX2, NOX4, Bax, Caspase-3, matrix metallopeptidase (MMP)2, MMP9,collagen I, Beclin-1, brain natriuretic peptide (BNP), atrial natriuretic peptide (ANP), $\beta$ myosin heavy chain $(\beta-\mathrm{MHC})$, growth differentiation factor 15 (GDF15), tropomyosin 1 (TPM1), biglycan (BGN) and periostin (POSTN) were significantly lower in the DOX+Val group, whereas the mRNA expression levels of BCL2 and collagen III were significantly higher in the DOX+Val group compared with the DOX group (all $\mathrm{P}<0.05$; Table II). Myocardial mRNA expression levels of NOX1, atrial natriuretic peptide-converting enzyme (CORIN) and transforming growth factor (TGF)- $\beta 1$ were similar between DOX and DOX+Val groups. The results suggested that the simultaneous application of Val with DOX could reduce the DOX-induced myocardial mRNA expressions of various NAD $(\mathrm{P}) \mathrm{H}$ oxidase, apoptosis and collagen signaling molecules, and increase the mRNA expression levels of anti-apoptotic signaling molecule in rats.

Effect of DOX+Val on myocardial protein expression levels of NOX1, NOX 2 and NOX4. Myocardial protein expression levels of NOX1, NOX2 and NOX4 were significantly higher in the DOX treated group compared with the CON group, whereas the expression levels were significantly lower in the DOX+Val group compared with the DOX group (all $\mathrm{P}<0.05$; Fig. 4C). The results indicated that simultaneous application of Val with DOX resulted in a reduction of DOX-induced myocardial protein expression levels of NOX1, NOX2 and NOX4. Myocardial levels of renin, ALD, TNF- $\alpha$, IL-6, BNP, ROS, MDA, SOD, NOX1, NOX2 and NOX4 were measured using ELISA (Table III). Myocardial levels of renin, ALD, TNF- $\alpha$, IL-6, BNP, ROS, MDA, NOX1, NOX2 and NOX4 were significantly higher in the DOX group compared with the CON group (all $\mathrm{P}<0.05$ ), and the levels of these factors were significantly lower in the DOX+Val group compared with the DOX group (all $\mathrm{P}<0.05$ ). Myocardial SOD levels were significantly lower in the DOX group compared with the CON group, and significantly higher in the DOX+Val group compared with the DOX group (both $\mathrm{P}<0.05$ ). These results suggest that simultaneous application of Val with DOX decreased DOX-induced changes in the myocardial levels of renin, ALD, TNF- $\alpha$, IL-6, BNP, ROS, MDA, NOX1, NOX2 and NOX4 and increased SOD levels.

Effect of DOX+Val on cell viability. $\mathrm{H} 9 \mathrm{C} 2$ cells were treated with various concentrations of DOX $(0.1-50 \mu \mathrm{M})$ for $24 \mathrm{~h}$. DOX treatment resulted in decreased cell viability in a dose-dependent manner, and cell viability was significantly decreased by $1 \mu \mathrm{M}$ DOX treatment. Thus, $1 \mu \mathrm{M}$ DOX was 

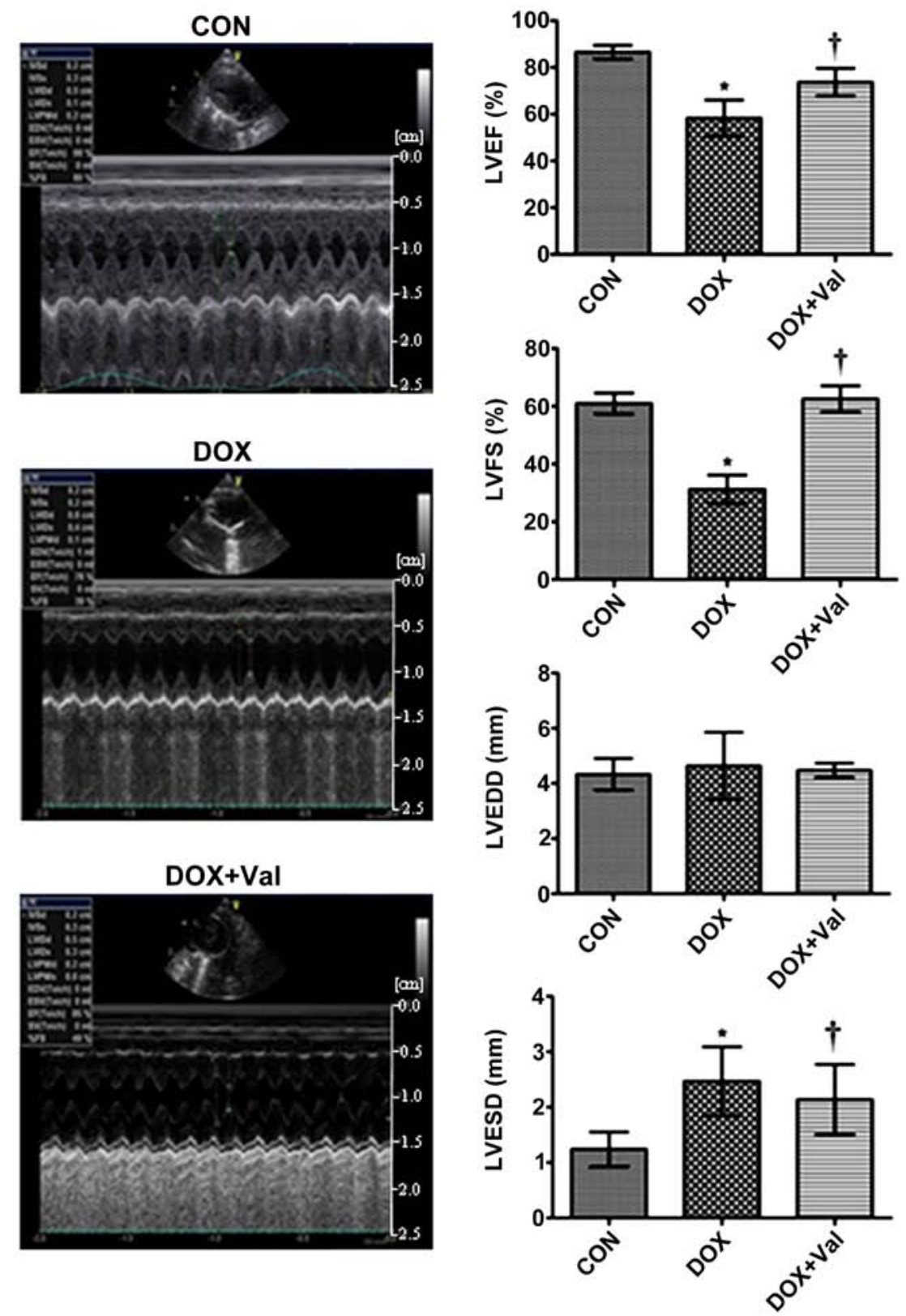

Figure 3. Representative echocardiographic images and cardiac functional parameters. " $\mathrm{P}<0.05$ vs. CON; ${ }^{\dagger} \mathrm{P}<0.05$ vs. DOX. LVEF, left ventricular ejection fraction; LVFS, left ventricular fraction shortening; LVEDD, left ventricular end-diastolic diameter; LVESD, left ventricular end-systolic diameter; CON, control; DOX, doxorubicin; Val, valsartan.

chosen as the target dose for subsequent experiments (Fig. 5A). H9C2 cells were also treated with various concentrations of Val $(0.1-30 \mu \mathrm{M})$ for $24 \mathrm{~h}$, and the results showed that Val did not affect cell survival (Fig. 5B). As shown in Fig. 5C, DOX-induced H9C2 cytotoxicity was significantly attenuated after $1 \mathrm{~h}$ of pre-treatment with 5 and $10 \mu \mathrm{M} / 1 \mathrm{Val}$.

Verification of transfection efficacy of siRNA and OE vector. As presented in Fig. S1, NOX2 and NOX4 mRNA expressions in the negative siRNA group and Empty vector group were similar with the Control group. It was apparent that the designed siRNA, vector and transfection process do not affect the expression of the targeted gene in H9c2 cells. The expressions of NOX2 and NOX4 in NOX2 siRNA group and NOX4 siRNA groups were significantly lower compared with the negative siRNA group, and their expressions in the NOX2 OE group and NOX4 OE group were significantly higher compared with the Empty vector group. These results indicated that siRNA and OE vectors transfection is high effective.

Effect of DOX+Val on intracellular ROS production in vitro. As shown in Fig. 6A and B, intracellular ROS production was significantly increased in the DOX-treated $\mathrm{H} 9 \mathrm{C} 2$ cells compared with the CON group. ROS production was significantly reduced in the DOX+Val-treated $\mathrm{H} 9 \mathrm{C} 2$ cells compared with the DOX group. Similarly, intracellular ROS production in the DOX-treated $\mathrm{H} 9 \mathrm{C} 2$ cells was significantly reduced by knockdown of NOX2 and NOX4, and intracellular ROS production in the DOX-treated $\mathrm{H} 9 \mathrm{C} 2$ cells was further increased following NOX2 and NOX4 overexpression. Intracellular ROS 
Table II. mRNA expression of myocardial genes in CON (n=8), DOX (n=12) and DOX+Val (n=12) groups.

\begin{tabular}{|c|c|c|c|}
\hline Gene & $\mathrm{CON}($ mean $\pm \mathrm{SD})$ & $\mathrm{DOX}($ mean $\pm \mathrm{SD})$ & $\mathrm{DOX}+\mathrm{Val}($ mean $\pm \mathrm{SD})$ \\
\hline Bax & $0.005472 \pm 0.002241$ & $0.579347 \pm 0.015441^{\mathrm{a}}$ & $0.016487 \pm 0.002301^{\mathrm{a}, \mathrm{b}}$ \\
\hline Collagen I & $0.000159 \pm 0.000117$ & $0.003708 \pm 0.001421^{\mathrm{a}}$ & $0.000743 \pm 0.000246^{\mathrm{a}, \mathrm{b}}$ \\
\hline BNP & $0.008863 \pm 0.000374$ & $0.033877 \pm 0.011124^{\mathrm{a}}$ & $0.015468 \pm 0.003612^{\mathrm{b}}$ \\
\hline Beclin-1 & $0.070218 \pm 0.038941$ & $0.496883 \pm 0.027112^{\mathrm{a}}$ & $0.408002 \pm 0.014891^{\mathrm{a}, \mathrm{b}}$ \\
\hline BCL2 & $0.069447 \pm 0.024328$ & $0.005516 \pm 0.002308^{a}$ & $0.011095 \pm 0.006306^{\mathrm{b}}$ \\
\hline NOX4 & $2.660319 \pm 0.722017$ & $23.744164 \pm 9.831247^{\mathrm{a}}$ & $4.283176 \pm 0.274911^{\mathrm{a}, \mathrm{b}}$ \\
\hline NOX1 & $0.329351 \pm 0.027661$ & $0.715372 \pm 0.196175^{\mathrm{a}}$ & $0.616009 \pm 0.201272$ \\
\hline NOX2 & $0.005916 \pm 0.002794$ & $0.085991 \pm 0.008259^{a}$ & $0.008974 \pm 0.000806^{\mathrm{a}, \mathrm{b}}$ \\
\hline Caspase-3 & $0.000813 \pm 0.000582$ & $0.004873 \pm 0.003057^{\mathrm{a}}$ & $0.002485 \pm 0.001034^{\mathrm{a}, \mathrm{b}}$ \\
\hline MMP9 & $0.000110 \pm 0.000222$ & $0.001814 \pm 0.000453^{\mathrm{a}}$ & $0.000134 \pm 0.000177^{\mathrm{a}, \mathrm{b}}$ \\
\hline MMP2 & $0.106209 \pm 0.082461$ & $0.409883 \pm 0.172409^{a}$ & $0.160207 \pm 0.036443^{\mathrm{a}, \mathrm{b}}$ \\
\hline ANP & $1.263391 \pm 0.404303$ & $7.823333 \pm 2.880115^{a}$ & $2.786022 \pm 0.582717^{\mathrm{a}, \mathrm{b}}$ \\
\hline$\beta-\mathrm{MHC}$ & $1.473521 \pm 0.610019$ & $24.508593 \pm 9.604317^{\mathrm{a}}$ & $3.712543 \pm 1.140049^{\mathrm{a}, \mathrm{b}}$ \\
\hline BGN & $3.914408 \pm 0.593123$ & $0.594062 \pm 0.097032^{\mathrm{a}}$ & $0.038563 \pm 0.004427^{\mathrm{a}, \mathrm{b}}$ \\
\hline TPM1 & $0.007855 \pm 0.003824$ & $0.002717 \pm 0.001296^{\mathrm{a}}$ & $0.000702 \pm 0.000241^{\mathrm{a}, \mathrm{b}}$ \\
\hline Collagen III & $0.017781 \pm 0.003225$ & $0.000461 \pm 0.000158^{\mathrm{a}}$ & $0.001982 \pm 0.000791^{\mathrm{a}, \mathrm{b}}$ \\
\hline CORIN & $0.245495 \pm 0.074003$ & $0.030678 \pm 0.028541^{\mathrm{a}}$ & $0.016321 \pm 0.008023^{\mathrm{a}}$ \\
\hline TGF- $\beta 1$ & $0.013657 \pm 0.004844$ & $0.039625 \pm 0.011513^{a}$ & $0.022389 \pm 0.012937$ \\
\hline GDF15 & $0.003563 \pm 0.001879$ & $0.044461 \pm 0.013455^{\mathrm{a}}$ & $0.004693 \pm 0.000264^{\mathrm{a}, \mathrm{b}}$ \\
\hline POSTN & $0.003533 \pm 0.002247$ & $0.530036 \pm 0.110447^{a}$ & $0.020532 \pm 0.006552^{\mathrm{a}, \mathrm{b}}$ \\
\hline
\end{tabular}

${ }^{\mathrm{a} P}<0.05$ vs. CON group; ${ }^{\mathrm{b}} \mathrm{P}<0.05$ vs. DOX group. CON, control; DOX, doxorubicin; Val, valsartan; NOX, NAD(P)H oxidase; BNP, brain natriuretic peptide; MMP, matrix metallopeptidase; ANP, atrial natriuretic peptide; $\beta$-MHC, $\beta$ myosin heavy chain; GDF15, growth differentiation factor 15; TPM1, tropomyosin 1; BGN, biglycan; POSTN, periostin; CORIN, atrial natriuretic peptide-converting enzyme; TGF- $\beta 1$, transforming growth factor $\beta 1$.

Table III. Myocardial content of genes in CON $(n=8)$, DOX $(n=12)$ and DOX+Val $(n=12)$ groups.

\begin{tabular}{lccc}
\hline Protein, $\mathrm{ng} / \mathrm{ml}$ & CON $($ mean $\pm \mathrm{SD})$ & DOX $($ mean $\pm \mathrm{SD})$ & DOX+Val $($ mean \pm SD $)$ \\
\hline Renin & $27.27 \pm 2.32$ & $41.82 \pm 3.76^{\mathrm{a}}$ & $34.02 \pm 3.14^{\mathrm{a}, \mathrm{b}}$ \\
ALD & $246.55 \pm 68.03$ & $376.87 \pm 85.34^{\mathrm{a}}$ & $264.25 \pm 71.05^{\mathrm{b}}$ \\
MDA & $2.55 \pm 0.72$ & $3.99 \pm 0.93^{\mathrm{a}}$ & $2.72 \pm 0.77^{\mathrm{b}}$ \\
SOD & $144.61 \pm 32.15$ & $105.48 \pm 22.65^{\mathrm{a}}$ & $138.84 \pm 18.16^{\mathrm{b}}$ \\
ROS & $275.42 \pm 71.86$ & $355.64 \pm 100.17^{\mathrm{a}}$ & $327.41 \pm 69.88^{\mathrm{b}}$ \\
TNF- $\alpha$ & $173.52 \pm 20.88$ & $260.24 \pm 23.47^{\mathrm{a}}$ & $211.77 \pm 20.59^{\mathrm{b}}$ \\
IL-6 & $77.09 \pm 17.26$ & $88.28 \pm 21.73^{\mathrm{a}}$ & $73.27 \pm 15.15^{\mathrm{b}}$ \\
BNP & $208.31 \pm 33.62$ & $331.77 \pm 22.45^{\mathrm{a}}$ & $275.73 \pm 26.21^{\mathrm{a}, \mathrm{b}}$ \\
NOX1 & $59.77 \pm 8.94$ & $68.87 \pm 10.16^{\mathrm{a}}$ & $59.13 \pm 7.65^{\mathrm{b}}$ \\
NOX2 & $44.22 \pm 9.55$ & $51.41 \pm 7.31^{\mathrm{a}}$ & $49.21 \pm 3.80^{\mathrm{b}}$ \\
NOX4 & $21.76 \pm 5.76$ & $27.37 \pm 6.41^{\mathrm{a}}$ & $21.08 \pm 5.49^{\mathrm{b}}$ \\
\hline
\end{tabular}

${ }^{\mathrm{a}} \mathrm{P}<0.05$ vs. CON group; ${ }^{\mathrm{b}} \mathrm{P}<0.05$ vs. DOX group. CON, control; DOX, doxorubicin; Val, valsartan; NOX, NAD(P)H oxidase; SOD, superoxide dismutase; ROS, reactive oxygen species; BNP, brain natriuretic peptide; ALD, aldosterone; MDA, malondialdehyde.

production in DOX-treated H9C2 cells was not significantly affected by the control siRNA or empty vector transfection.

Effect of DOX+Val on cardiomyocyte apoptosis. As shown in Fig. 7A and B, cardiomyocyte apoptosis was significantly increased in the DOX-treated H9C2 cells compared with the CON group. Apoptosis was significantly reduced by DOX+Val treatment, as well as NOX2 and NOX4 knockdown. Apoptosis rates in the DOX-treated $\mathrm{H} 9 \mathrm{C} 2$ cells were further increased following NOX2 and NOX4 overexpression. Cardiomyocyte 
A

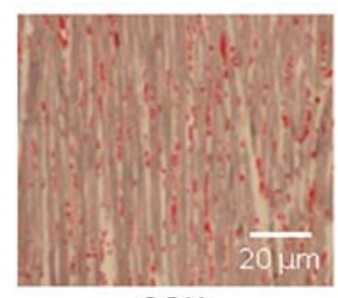

CON

B

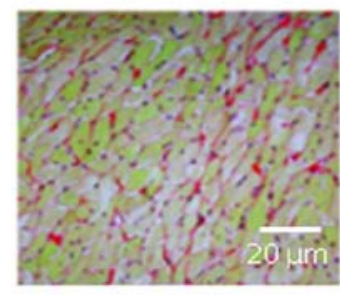

CON

C

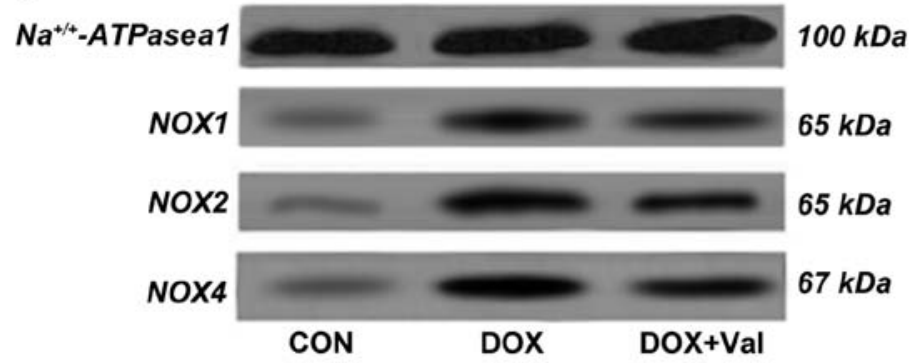

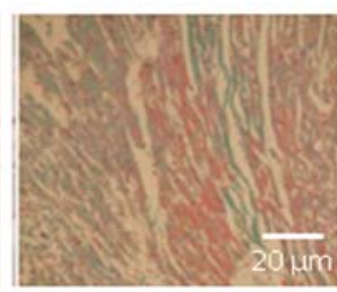

DOX

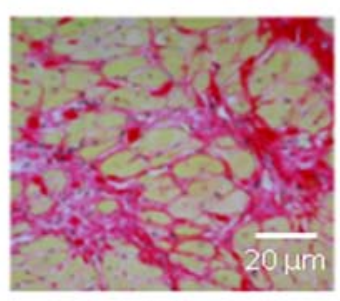

DOX
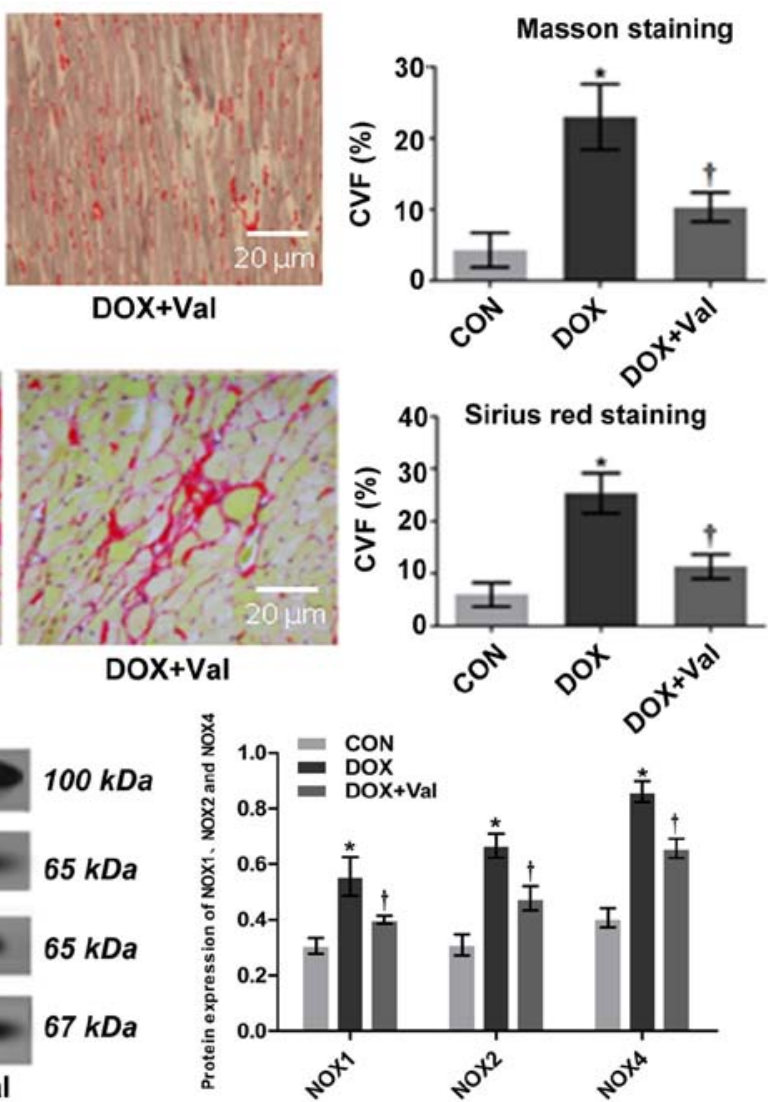

Figure 4. Myocardial histopathology and protein expression of NOX1, NOX2 and NOX4. (A) Masson staining and CVF (magnification, x200). (B) Sirius red staining and CVF (magnification, x200). Collagen stained with Masson staining (blue) and Sirius red staining (red) are significantly increased in the DOX group compared with those in the CON group, which is attenuated in the DOX+Val group. (C) Myocardial protein expression of NOX1, NOX2 and NOX4 detected by western blotting. ${ }^{*} \mathrm{P}<0.05$ vs. CON; ${ }^{\dagger} \mathrm{P}<0.05$ vs. DOX.CON, control; DOX, doxorubicin; Val, valsartan; NOX, NAD(P)H oxidase; CVF, collagen volume fraction.

A

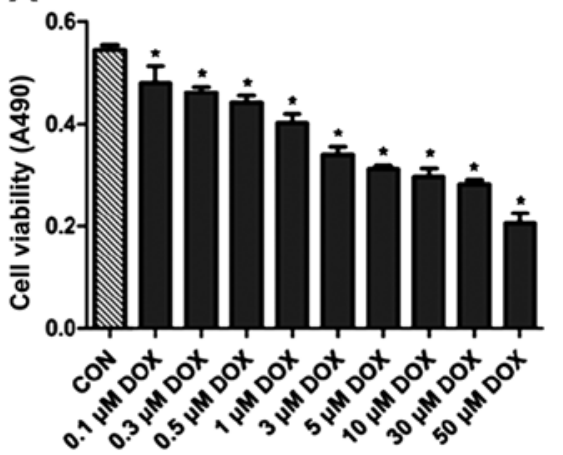

B

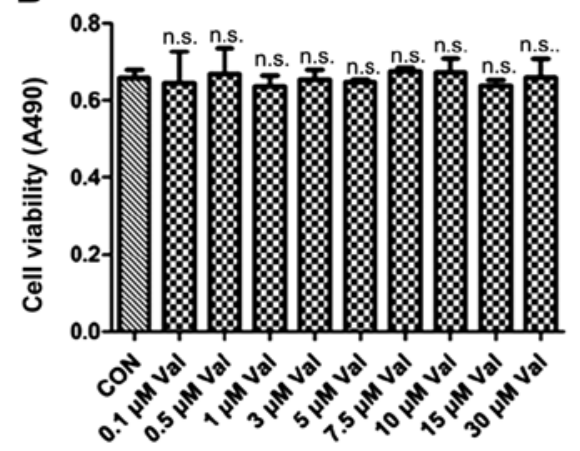

C

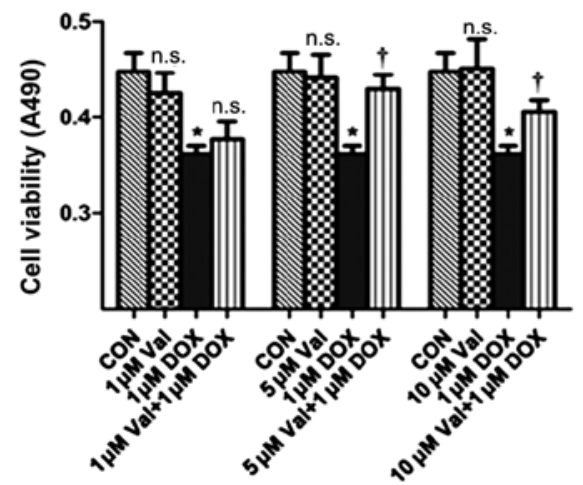

Figure 5. Cell viability measured by MTT assay. (A) H9C2 cells were treated with various concentrations of DOX for $24 \mathrm{~h}$, DOX resulted in cell death in a dose-dependent manner. Cell viability significantly decreased with $1 \mu \mathrm{M} \mathrm{DOX}$, so this concentration was chosen as the target dose. (B) H9C2 cells were treated with numerous concentrations of Val, the results revealed that the nine different concentrations of Val did not affect the survival of H9C2 cells within $24 \mathrm{~h}$. (C) Val concentration required to protect against DOX-induced cytotoxicity was calculated by performing a dose-response study in the presence of 1,5 and $10 \mu \mathrm{M}$ Val. The cytotoxic effects of DOX were significantly attenuated by pre-treatment with 5 and $10 \mu \mathrm{M}$ Val. Based on these results, $5 \mu \mathrm{M}$ Val was selected as the target dose for further study. "P<0.05 vs. CON; 'P $<0.05$ vs. DOX; n.s., not significant. CON, control; DOX, doxorubicin; Val, valsartan; NOX, NAD(P)H oxidase.

apoptosis in the DOX-treated H9C2 cells was not affected by the control siRNA or empty vector transfection.

\section{Discussion}

The results of the present study showed that a combined treatment of Val and DOX significantly attenuated DOX-induced myocardial injury and reduced DOX-induced ROS production and apoptosis, potentially via downregulation of the NOX2/NOX4 signaling pathway.

In agreement with previous studies (7,33-35), it was shown that simultaneous application of Val with DOX attenuated DOX-induced myocardial injury in rats, as shown by the improvement in cardiac function and reduction in 


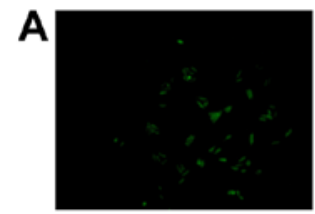

Control

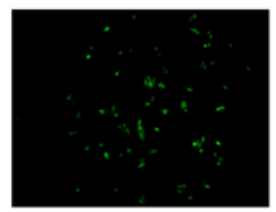

DOX+NOX4 siRNA

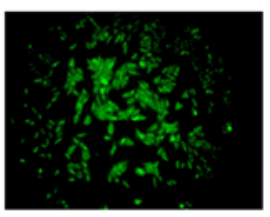

DOX

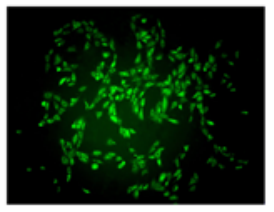

DOX + Negative siRNA

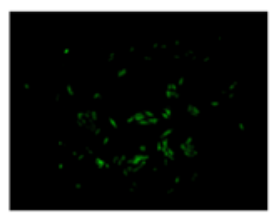

Val

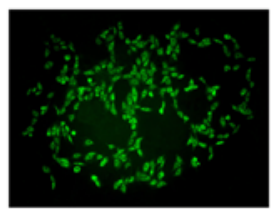

DOX+Empty vector

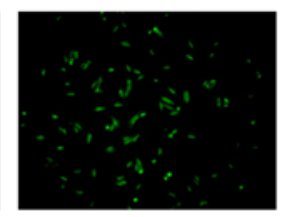

DOX+Val

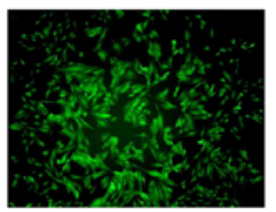

DOX+NOX2 OE

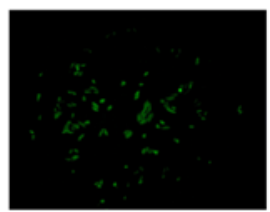

DOX+NOX2 siRNA

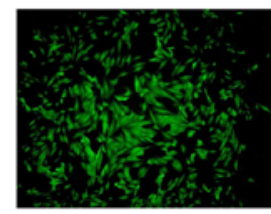

DOX+NOX4 OE

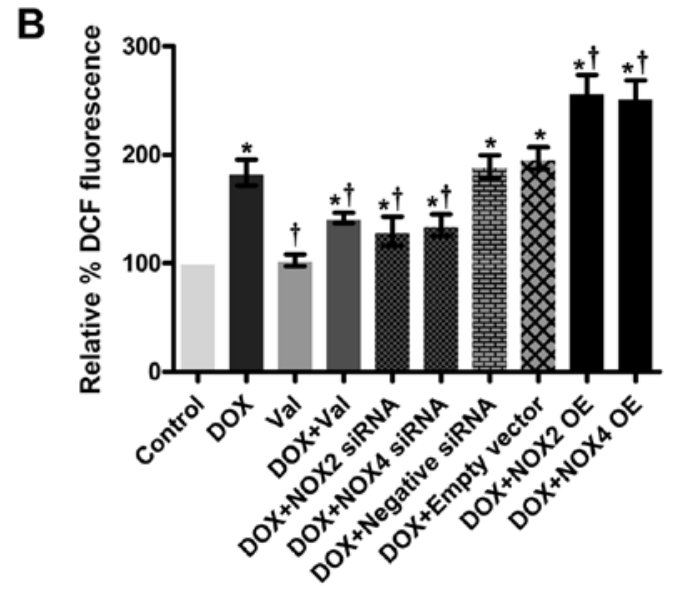

Figure 6. Intracellular ROS production in vitro in $\mathrm{H} 9 \mathrm{C} 2$ cells post-treatment. (A) Expression of ROS in $\mathrm{H} 9 \mathrm{C} 2$ cells was detected by a DCF-DA assay under fluorescence microscope (magnification, $\mathrm{x} 100$ ). $\mathrm{H} 9 \mathrm{C} 2$ cells were treated as follows: i) Control group; ii) $\mathrm{H} 9 \mathrm{C} 2$ cells exposed to $1 \mu \mathrm{M} \mathrm{DOX}$ for $24 \mathrm{~h}$; iii) cells exposed to $5 \mu \mathrm{M}$ Val for $1 \mathrm{~h}$; iv) cells were pre-treated with $5 \mu \mathrm{M}$ Val for $1 \mathrm{~h}$ followed by treatment with $1 \mu \mathrm{M}$ DOX for $24 \mathrm{~h}$; v) cells were transiently transfected with NOX2-siRNA for $24 \mathrm{~h}$ followed by treatment with $1 \mu \mathrm{M}$ DOX for $24 \mathrm{~h}$; vi) cells were transiently transfected with NOX4-siRNA for $24 \mathrm{~h}$ followed by treatment with $1 \mu \mathrm{M}$ DOX for $24 \mathrm{~h}$; vii) cells were transiently transfected with negative siRNA for $24 \mathrm{~h}$ followed by treatment with $1 \mu \mathrm{M}$ DOX for $24 \mathrm{~h}$; viii) cells were transiently transfected with an empty vector for $24 \mathrm{~h}$; ix) NOX2-OE cells were treated with $1 \mu \mathrm{M}$ DOX for $24 \mathrm{~h} ; \mathrm{x}$ ) NOX4-OE cells were treated with $1 \mu \mathrm{M}$ DOX for $24 \mathrm{~h}$. (B) Semi-quantitative analysis of ROS production detected by DCF-DA. " $\mathrm{P}<0.05$ vs. control; ' $\mathrm{P}<0.05$ vs. DOX. DCF-DA, dichlorofluorescein; ROS, reactive oxygen species; DOX, doxorubicin; Val, valsartan; NOX, NAD(P)H oxidase; siRNA, small interfering RNA; OE, overexpressed.

cardiac remodeling. Compared with the CON group, the CVF was significantly increased, along with upregulated expression of MMP2, MMP9 and collagen I in the DOX group, suggesting enhanced fibrosis was responsible for reduced cardiac function in this model. Treatment with Val and DOX significantly reversed the aforementioned changes induced by DOX, suggesting that Val protected the rats from DOX-induced myocardial injury by reducing collagen remolding and myocardial fibrosis, possibly by downregulating myocardial expression of MMP2, MMP9 and collagen I. This result is in agreement with a previous study that demonstrated that co-administration of telmisartan with DOX decreased the levels of cardiotoxicity-associated biochemical markers (lactate dehydrogenase and creatine kinase myocardial band) and attenuated the effects of DOX on oxidative stress parameters and nitric oxide production, as well as myocardial fibrosis (36). Taken together, these results suggest that application of ARBs with DOX at the time of drug initiation may be a clinically feasible strategy to prevent/attenuate the potential cardiac damage induced by DOX in patients with tumors.

Increased ROS production and reduced SOD levels are frequently observed pathological features of various cardiovascular diseases, such as atherosclerosis and hypertension $(37,38)$. In the present study, MDA levels were increased and SOD levels were decreased in myocardial tissues from the DOX-treated rats in vivo, and increased ROS production and apoptosis were observed in the DOX-treated $\mathrm{H} 9 \mathrm{C} 2$ cells in vitro. Similarly, simultaneous treatment with Val significantly reversed these changes both in vivo and in vitro. Furthermore, it was shown that mRNA expression levels of the pro-apoptotic genes Caspase- 3 and Bax were upregulated, whereas the mRNA expression levels of BCL2 were downregulated in the DOX group, and Val treatment reversed these changes. Taken together, these results suggest that treatment with Val may effectively reduce the enhanced myocardial apoptosis induced by DOX, possibly through modulating the expression of apoptosis-related genes. Additionally, these results suggest that the beneficial effects of Val in this model are at least partly associated with the capacity of Val to reduce DOX-induced myocardial injury via the reduction of myocardial apoptosis.

The myocardial mRNA and protein expression levels of NOX1, NOX2 and NOX4 following DOX treatment were measured. The results showed that DOX treatment significantly upregulated the myocardial protein expression of 


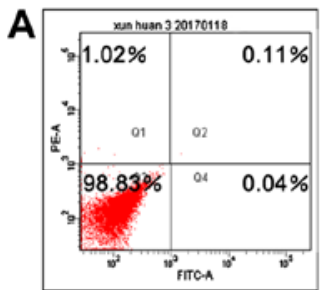

Control

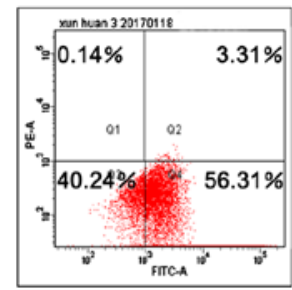

DOX+NOX4 SIRNA

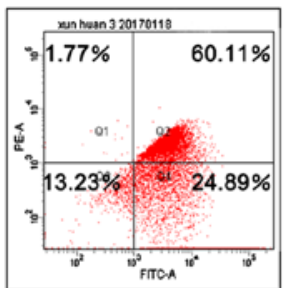

DOX

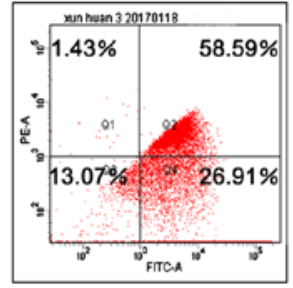

DOX+Negative siRNA

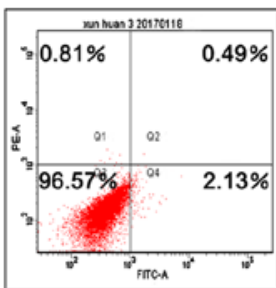

Val

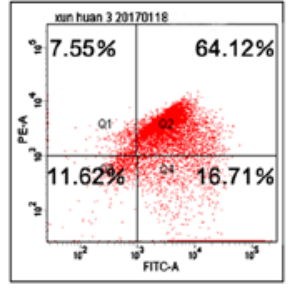

DOX+Empty vector

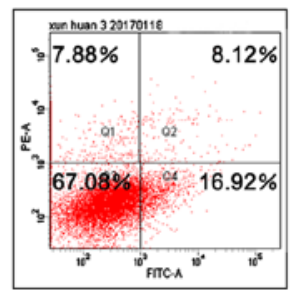

DOX + Val

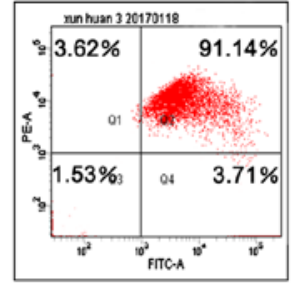

DOX+ NOX2 OE

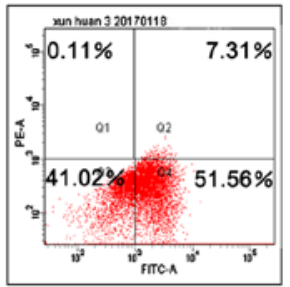

DOX+NOX2 SIRNA

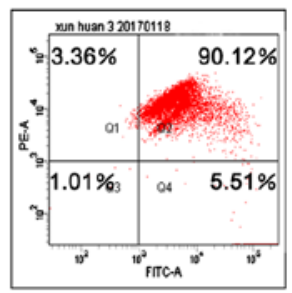

DOX+NOX4 OE

B

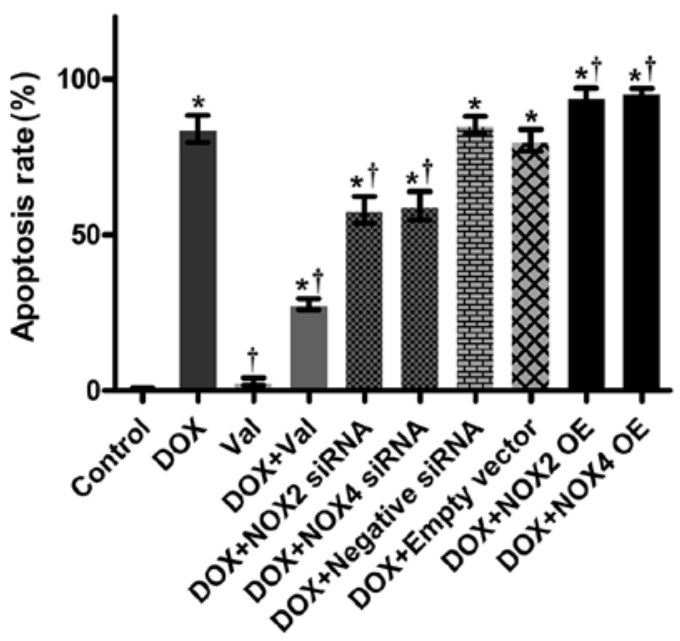

Figure 7. Apoptosis in vitro in $\mathrm{H} 9 \mathrm{C} 2$ cells post-treatment. (A) Apoptosis in $\mathrm{H} 9 \mathrm{C} 2$ cells detected by flow cytometry. (B) Cardiomyocyte apoptosis was significantly increased in DOX-treated H9C2 cells compared with the control group, which was significantly reduced by DOX+Val and NOX2-siRNA and NOX4-siRNA, whereas apoptosis in DOX-treated $\mathrm{H} 9 \mathrm{C} 2$ cells was further increased following NOX2 and NOX4 overexpression. ${ }^{*} \mathrm{P}<0.05$ vs. control; ${ }^{\dagger} \mathrm{P}<0.05$ vs. DOX. DOX, doxorubicin; Val, valsartan; NOX, NAD(P)H oxidase; siRNA, small interfering RNA; OE, overexpressed.

NOX1, NOX2 and NOX4, and mRNA expression of NOX2 and NOX4. Simultaneous treatment with Val significantly reduced the mRNA expression levels of NOX2 and NOX4, as well as the myocardial protein expression levels of NOX1, NOX2 and NOX4. These results suggest that upregulated NOX expression levels, particularly NOX 2 and NOX4 signaling, may contribute to DOX-induced cardiac injury, and the observed beneficial effects of simultaneous application of Val may partially be associated with its capacity to downregulate NOX2 and NOX4 signaling in this model.

To explore the mechanism underlying the protective effects of Val in DOX-induced myocardial injury, ROS production and apoptosis of DOX-treated $\mathrm{H} 9 \mathrm{C} 2$ cells were observed following up- or downregulation of NOX2 and NOX4 expression, as Val significantly downregulated the myocardial mRNA expression of these two genes. The results suggested that ROS production and apoptosis were significantly reduced by downregulating NOX2 and NOX4, whereas overexpression of NOX2 and NOX4 increased ROS production and apoptosis in DOX-treated
H9C2 cells, suggesting that downregulation of NOX2 and NOX4 expression may be mechanism by which Val alleviates DOX-induced myocardial injury. Although the present study observed changes in intracellular ROS and cardiomyocyte apoptosis under the conditions of NOX-overexpressing and NOX-silencing, we did not detect them at the protein level, which is a limitation of this study. In our previous study examining the mechanisms of DOX toxicity, the expression of angiotensin (Ang)II receptor protein (AT1R) was significantly upregulated following DOX stimulation in $\mathrm{H} 9 \mathrm{C} 2$ cells, and AngII increased the protein expression levels of NOX2 and NOX4 and the production of ROS (data not yet published). The protein expression levels of ERK, JNK and P38, which lie downstream of the mitogen-activated protein kinase (MAPK) signaling pathway, were increased significantly (data not yet published). Pre-treatment with Val reduced the expression of AT1R, NOX2, NOX4 and ROS, and activity of the MAPK signaling pathway was decreased (data not yet published). Thus, it was hypothesized that the AngII-NOX-ROS-MAPK 
signaling pathway may underlie DOX-induced myocardial toxicity.

In summary, co-treatment with Val and DOX significantly reduced DOX-induced myocardial injury, potentially through downregulation of NOX2 and NOX4 signaling. The present study provides experimental evidence supporting the simultaneous use of ARBs to prevent DOX-induced cardiotoxicity in the clinical setting.

\section{Acknowledgements}

Not applicable.

\section{Funding}

The present study was supported by the Natural Science Funds of Liaoning Province (grant no. 201602033) and the National Natural Science Foundation of China (grant no. 81770405).

\section{Availability of data and materials}

The datasets used and/or analyzed during the current study are available from the corresponding author on reasonable request.

\section{Authors' contributions}

DC, LC and WT performed the experiments and data analysis, and prepared the manuscript. HW and QW contributed to the conception and design of the study and critically revised the manuscript for important intellectual content and supervised the experimental process. LM and ZL prepared the figures, and analyzed and interpreted the data. QY designed this study and proofread the manuscript. All authors read and approved the final manuscript.

\section{Ethics approval and consent to participate}

All experiments were performed in compliance with the ARRIVE guidelines as well as the Guide for the Care and Use of Laboratory Animals of the National Academy of Sciences. The Institutional Animal Research and Ethics Committee of Dalian University approved the protocols of the animal experiments.

\section{Patient consent for publication}

Not applicable.

\section{Competing interests}

The authors declare that they have no competing interests.

\section{References}

1. Razavi-Azarkhiavi K, Iranshahy M, Sahebkar A, Shirani K and Karimi G: The protective role of phenolic compounds against doxorubicin-induced cardiotoxicity: A comprehensive review. Nutr Cancer 68: 892-917, 2016.

2. Swain SM, Whaley FS and Ewer MS: Congestive heart failure in patients treated with doxorubicin: A retrospective analysis of three trials. Cancer 97: 2869-2879, 2003.
3. Ganame J, Claus P, Uyttebroeck A, Renard M, D'hooge J, Bijnens B, Sutherland GR, Eyskens B and Mertens L: Myocardial dysfunction late after low-dose anthracycline treatment in asymptomatic pediatric patients. J Am Soc Echocardiogr 20: 1351-1358, 2007.

4. Tebbi CK, London WB, Friedman D, Villaluna D, De Alarcon PA Constine LS, Mendenhall NP, Sposto R, Chauvenet A and Schwartz CL: Dexrazoxane-associated risk for acute myeloid leukemia/myelodysplastic syndrome and other secondary malignancies in pediatric Hodgkin's disease. J Clin Oncol 25: 493-500, 2007.

5. Shaikh AY and Shih JA: Chemotherapy-induced cardiotoxicity. Curr Heart Fail Rep 9: 117-127, 2012.

6. Iqbal M, Dubey K, Anwer T, Ashish A and Pillai KK: Protective effects of telmisartan against acute doxorubicin-induced cardiotoxicity in rats. Pharmacol Rep 60: 382-390, 2008.

7. Zong WN, Yang XH, Chen XM, Huang HJ, Zheng HJ, Qin XY, Yong YH, Cao K, Huang J and Lu XZ: Regulation of angiotensin-(1-7) and angiotensin II type 1 receptor by telmisartan and losartan in adriamycin-induced rat heart failure. Acta Pharmacol Sin 32: 1345-1350, 2011.

8. Angsutararux P,Luanpitpong S and Issaragrisil S: Chemotherapyinduced cardiotoxicity: Overview of the roles of oxidative stress. Oxid Med Cell Longev 2015: 795602, 2015.

9. Chang SA, Lim BK, Lee YJ, Hong MK, Choi JO and Jeon ES: A Novel Angiotensin Type I receptor antagonist, fimasartan, prevents doxorubicin-induced cardiotoxicity in rats. J Korean Med Sci 30: 559-568, 2015.

10. Manohar P and Pina IL: Therapeutic role of angiotensin II receptor blockers in the treatment of heart failure. Mayo Clin Proc 78: 334-338, 2003.

11. Nakamae H, Tsumura K, Terada Y, Nakane T, Nakamae M, Ohta K, Yamane T and Hino M: Notable effects of angiotensin II receptor blocker, valsartan, on acute cardiotoxic changes after standard chemotherapy with cyclophosphamide, doxorubicin, vincristine, and prednisolone. Cancer 104: 2492-2498, 2005.

12. Sakr HF, Abbas AM and Elsamanoudy AZ: Effect of valsartan on cardiac senescence and apoptosis in a rat model of cardiotoxicity. Can J Physiol Pharmacol 94: 588-598, 2016.

13. Chen L, Yan KP, Liu XC, Wang W, Li C, Li M and Qiu CG: Valsartan regulates TGF- $\beta /$ Smads and TGF- $\beta / p 38$ pathways through IncRNA CHRF to improve doxorubicin-induced heart failure. Arch Pharm Res 41: 101-109, 2018.

14. Hafstad AD, Nabeebaccus AA and Shah AM: Novel aspects of ROS signalling in heart failure. Basic Res Cardiol 108: 359, 2013.

15. Dietl A and Maack C: Targeting mitochondrial calcium handling and reactive oxygen species in heart failure. Curr Heart Fail Rep 14: 338-349, 2017.

16. Meitzler JL, Antony S, Wu Y, Juhasz A, Liu H, Jiang G, Lu J, Roy K and Doroshow JH: NADPH oxidases: A perspective on reactive oxygen species production in tumor biology. Antioxid Redox Signal 20: 2873-2889, 2014.

17. Bedard K and Krause KH: The NOX family of ROS-generating NADPH oxidases: Physiology and pathophysiology. Physiol Rev 87: 245-313, 2007.

18. Tsutsui H, Kinugawa $\mathrm{S}$ and Matsushima S: Oxidative stress and heart failure. Am J Physiol Heart Circ Physiol 301: H2181-H2190, 2011.

19. Zhao QD, Viswanadhapalli S, Williams P, Shi Q, Tan C, Yi X, Bhandari B and Abboud HE: NADPH oxidase 4 induces cardiac fibrosis and hypertrophy through activating Akt/mTOR and NFkB signaling pathways. Circulation 131: 643-655, 2015.

20. Ruzicka M, Yuan B, Harmsen E and Leenen FH: The renin-angiotensin system and volume overload-induced cardiac hypertrophy in rats. Effects of angiotensin converting enzyme inhibitor versus angiotensin II receptor blocker. Circulation 87: 921-930, 1993.

21. Bendall JK, Cave AC, Heymes C, Gall N and Shah AM: Pivotal role of a gp91(phox)-containing NADPH oxidase in angiotensin II-induced cardiac hypertrophy in mice. Circulation 105: 293-296, 2002.

22. Murdoch CE, Zhang M, Cave AC and Shah AM: NADPH oxidase-dependent redox signalling in cardiac hypertrophy, remodelling and failure. Cardiovasc Res 71: 208-215, 2006.

23. Seddon M, Looi YH and Shah AM: Oxidative stress and redox signalling in cardiac hypertrophy and heart failure. Heart 93: 903-907, 2007.

24. Watson LE, Sheth M, Denyer RF and Dostal DE: Baseline echocardiographic values for adult male rats. J Am Soc Echocardiogr 17: 161-167, 2004. 
25. Barauna VG, Rosa KT, Irigoyen MC and de Oliveira EM: Effects of resistance training on ventricular function and hypertrophy in a rat model. Clin Med Res 5: 114-120, 2007.

26. Liu HN, Wang HR, Cheng D, Pei ZW, Zhu N and Yu Q: Potential role of a disintegrin and metalloproteinase-17 (ADAM17) in age-associated ventricular remodeling of rats. RSC Advances 9: 14321-14330, 2019

27. Livak KJ and Schmittgen TD: Analysis of relative gene expression data using real-time quantitative PCR and the 2(-Delta Delta C(T)) method. Methods 25: 402-408, 2001.

28. Ye L, Haider HKH, Jiang S, Ge R, Law PK and Sim EK: In vitro functional assessment of human skeletal myoblasts after transduction with adenoviral bicistronic vector carrying human VEGF165 and angiopoietin-1. J Heart Lung Transplant 24: 1393-1402, 2005.

29. Xie F, Wu D, Huang SF, Cao JG, Li HN, He L, Liu MQ, Li LF and Chen LX: The endoplasmic reticulum stress-autophagy pathway is involved in apelin-13-induced cardiomyocyte hypertrophy in vitro. Acta Pharmacol Sin 38: 1589-1600, 2017

30. Huang YL, Zhao F, Luo CC, Zhang X, Si Y, Sun Z, Zhang L, Li QZ and Gao XJ: SOCS3-mediated blockade reveals major contribution of JAK2/STAT5 signaling pathway to lactation and proliferation of dairy cow mammary epithelial cells in vitro. Molecules 18: 12987-13002, 2013.

31. Priya LB, Baskaran R, Huang CY and Padma VV: Neferine ameliorates cardiomyoblast apoptosis induced by doxorubicin: Possible role in modulating NADPH oxidase/ROS-mediated NFkB redox signaling cascade. Sci Rep 7: 12283, 2017.

32. Gao J, Chen T, Zhao D, Zheng J and Liu Z: Ginkgolide B Exerts Cardioprotective properties against doxorubicin-induced cardiotoxicity by regulating reactive oxygen species, Akt and calcium signaling pathways in vitro and in vivo. PLoS One 11: e0168219, 2016.
33. Yu Q, Li Q, Na R, Li X, Liu B, Meng L, Liutong H, Fang W, Zhu $\mathrm{N}$ and Zheng $\mathrm{X}$ : Impact of repeated intravenous bone marrow mesenchymal stem cells infusion on myocardial collagen network remodeling in a rat model of doxorubicin-induced dilated cardiomyopathy. Mol Cell Biochem 387: 279-285, 2014.

34. Bakhtiari E, Hosseini A and Mousavi SH: Protective effect of Hibiscus sabdariffa against serum/glucose deprivation-induced PC12 cells injury. Avicenna J Phytomed 5: 231-237, 2015.

35. Huang CY, Chen JY, Kuo CH, Pai PY, Ho TJ, Chen TS Tsai FJ, Padma VV, Kuo WW, Huang CY, et al: Mitochondrial ROS-induced ERK1/2 activation and HSF2-mediated $\mathrm{AT}_{1} \mathrm{R}$ upregulation are required for doxorubicin-induced cardiotoxicity. J Cell Physiol 233: 463-475, 2018.

36. Ibrahim MA, Ashour OM, Ibrahim YF, El-Bitar HI, Gomaa W and Abdel-Rahim SR: Angiotensin-converting enzyme inhibition and angiotensin AT(1)-receptor antagonism equally improve doxorubicin-induced cardiotoxicity and nephrotoxicity. Pharmacol Res 60: 373-381, 2009.

37. Khosravi M, Poursaleh A, Ghasempour G, Farhad S and Najafi M: The effects of oxidative stress on the development of atherosclerosis. Biol Chem 400: 711-732, 2019.

38. Wu J, Saleh MA, Kirabo A, Itani HA, Montaniel KR, Xiao L, Chen W, Mernaugh RL, Cai H, Bernstein KE, et al: Immune activation caused by vascular oxidation promotes fibrosis and hypertension. J Clin Invest 126: 50-67, 2016. 